\title{
AN ANALYTICAL METHOD FOR WELL-FORMED WORKFLOW/PETRI NET VERIFICATION OF CLASSICAL SOUNDNESS
}

\author{
JULIO CLEMPNER
}

Center for Economics, Management and Social Research

National Polytechnic Institute, Lauro Aguirre 120, Col. Agricultura, Miguel Hidalgo, 11360, Mexico City, Mexico

e-mail: julio@clempner.name

\begin{abstract}
In this paper we consider workflow nets as dynamical systems governed by ordinary difference equations described by a particular class of Petri nets. Workflow nets are a formal model of business processes. Well-formed business processes correspond to sound workflow nets. Even if it seems necessary to require the soundness of workflow nets, there exist business processes with conditional behavior that will not necessarily satisfy the soundness property. In this sense, we propose an analytical method for showing that a workflow net satisfies the classical soundness property using a Petri net. To present our statement, we use Lyapunov stability theory to tackle the classical soundness verification problem for a class of dynamical systems described by Petri nets. This class of Petri nets allows a dynamical model representation that can be expressed in terms of difference equations. As a result, by applying Lyapunov theory, the classical soundness property for workflow nets is solved proving that the Petri net representation is stable. We show that a finite and non-blocking workflow net satisfies the sound property if and only if its corresponding $P N$ is stable, i.e., given the incidence matrix $A$ of the corresponding $\mathrm{PN}$, there exists a $\Phi$ strictly positive $m$ vector such that $A \Phi \leq 0$. The key contribution of the paper is the analytical method itself that satisfies part of the definition of the classical soundness requirements. The method is designed for practical applications, guarantees that anomalies can be detected without domain knowledge, and can be easily implemented into existing commercial systems that do not support the verification of workflows. The validity of the proposed method is successfully demonstrated by application examples.
\end{abstract}

Keywords: Petri nets, decidability, workflow nets, Lyapunov stability, soundness, verification.

\section{Introduction}

1.1. Brief review. A workflow model is put to use by feeding it to a workflow management system (zur Muehlen, 2004; Weske, 2007). The heart of a workflow management system is the workflow engine, which does the actual management (Mann, 2010). Workflow management systems are driven by business process models. Therefore, it is important to define and streamline business processes in order to improve efficiency and reduce operating cycle times. Ultimately, the success of such modeling efforts lies not only in careful technical design, but also in ensuring the well-formed business processes of such models. Effective business processes modeling involves understanding existing process defects, identifying sources of inefficiency (deadlocks, livelocks, and other anomalies), and redefining processes to increase efficiency or decrease errors. But workflow management systems do not support verification methods for business processes design (van der Aalst, 2011).
The success of workflow management systems and methodologies has been widely publicized, while the more serious failures have not. Mendling et al. (2007), based on more than 2000 process models including well-known sets of models, such as the SAP reference model, report that more than 10 percent of these models are awed.

Workflow nets were introduced by van der Aalst (1997; 1998) and are currently the most widely used model to formally describe workflow processes. Workflow nets are a formal model of business process responsible for the organization of the processing tasks. The existing graphical languages implemented by workflow management systems are typically token-based, and for this reason a transformation to Petri nets is reasonably simple.

Petri nets are a natural technique for formal modeling and analyzing workflow nets because the flow-oriented nature of workflow processes (Desel nad Erwin, 2000; 
Ellis and Nutt, 1993, van der Aalst, 1997; 1998). Petri nets are used for process representation, taking advantage of the well-known properties of this approach, namely, formal semantic and graphical display, giving a specific and unambiguous description of the behavior of the process. We consider workflow nets as dynamical systems governed by ordinary difference equations described by a particular class of Petri nets (Clempner and Retchkiman, 2005; Clempner, 2005).

Loosely speaking, a workflow net is a Petri net with an initial place and a distinguished final place called the sink. Well-formed business processes correspond to sound workflow nets (van der Aalst, 2007). Petri nets have been extensively studied since the mid 1990s as an abstraction of the workflow to check the soundness property (van der Aalst, 1998; 2007; 2011; Barkaoui and Ayed, 2011; Barkaoui and Petrucci, 1998; Basu and Blanning, 2000; 2002; Bi and Zhao, 2004; Clempner and Retchkiman, 2005; Clempner, 2014; Dehnert and Rittgen, 2001; van Dongen and Verbeek, 2005; Fu and Su, 2002; 2004; van Hee and Voorhoeve, 2005; 2004; Karamanolis and Wheater, 2000; Kindler and Reisig, 2000; Lin and Chen, 2002; Lohmann and Weinberg, 2006; Martens, 2005a; 2005b; Mendling and van der Aalst, 2007; Sadiq and Orłowska, 1997; 2000; Salimifard and Wright, 2001; Vanhatalo and Leymann, 2007; Verbeek and ter Hofstede, 2001, Verbeek and van der Aalst, 2001; Wombacher, 2006; Wynn and ter Hofstede, 2005; Wynn and Edmond, 2006). In their research the authors have proposed alternative notions of soundness and more sophisticated languages, making these notions undecidable.

For the length of the distinguished history and exciting life of Petri nets, research looks for an analytical method able to develop new fast and efficient techniques to solve any kind of problem. Petri nets are used as an abstraction of the workflow to check the soundness property. Even if it seems necessary to require the soundness of workflow nets, there exist business processes with conditional behavior that will not necessarily satisfy the soundness property. The problem is often not caused by the structure of the net, but by operations associated with transition labels that are being used. Then, given a Petri net, the computation can always be completed, that is, it is possible to show that a process initiated in the source place and, regardless of how the computation proceeds at the beginning, the Petri net has always a trajectory able to reach the sink place of the Petri net.

1.2. Main results. In this paper we propose an analytical method for showing that a workflow net satisfies the soundness property using a Petri net. The proposed analytical method guarantees that anomalies can be detected without domain knowledge. To present our statement, we use Lyapunov stability theory to tackle the soundness problem for a class of dynamical systems named discrete event systems, described by Petri nets. This class of Petri nets allows a dynamical model representation that can be expressed in terms of difference equations. As a result, by applying Lyapunov theory, the soundness property for workflow nets is solved showing that the Petri net representation is stable.

1.3. Organization of the paper. The remainder of this paper is organized as follows. We present some of the preliminaries including the mathematical notation and Petri nets basics in Section 2. In Section 3, we motivate the introduction of the soundness workflow verification technique, presenting the basic notion of workflow net and stability followed by the definition of soundness. We also describe and exemplify the finite and non-blocking conditions established for the Petri net. Section 4 outlines the core content of the paper, presenting the basic notions of stability and the main result of the paper about the soundness property. We present a formal approach showing how the soundness property can be computed over a finite and non-blocking workflow net. We also make emphasis on the reasons why the finite and non-blocking conditions cannot be relaxed. In Section 5 we present two examples which pragmatically illustrate the application of the method. Finally, in Section 6 some concluding remarks and future work are outlined.

\section{Preliminaries}

In this section, we present some well-established definitions and properties which will be used later (Brams, 1983). Let $\mathbb{N}=\{0,1,2, \ldots\}, \mathbb{N}_{+}^{n_{0}}=$ $\left\{n_{0}, n_{0}+1, \ldots, n_{0}+k, \ldots\right\}, n_{0} \geq 0, \mathbb{R}=(-\infty, \infty)$ and $\mathbb{R}_{+}=[0, \infty)$.

A (marked) Petri net is a quintuple $P N=$ $\left(P, Q, F, W, M_{0}\right)$, where $P=\left\{p_{1}, p_{2}, \ldots, p_{m}\right\}$ is a finite set of places, $Q=\left\{q_{1}, q_{2}, \ldots, q_{n}\right\}$ is a finite set of transitions with $P \cap Q=\emptyset$, and $P$ and $Q$ are nonempty such that $P \cup Q \neq \emptyset, F \subseteq(P \times Q) \cup(Q \times P)$ is a set of arcs which determines a flow relation, $W: F \rightarrow \mathbb{N}_{+}^{1}$ is a weight function, $M_{0}: P \rightarrow \mathbb{N}$ is the initial marking. We adopt the standard rules about representing nets as directed graphs, namely, places are represented as circles, transitions as rectangles, the flow relation by arcs, and markings are shown by placing tokens within circles. At any time a place contains zero or more tokens, drawn as black dots (Murata, 1989).

For each transition or place $z$ we will denote by $\bullet z:=\quad\{y \in P \cup Q \mid(y, z) \in F\}$ the preset of $z$. Analogously, we will denote by $z \bullet=\{y \in P \cup Q \mid(z, y) \in F\}$ the postset of $z$. A source place is a place $p_{0} \in P$ such that $\bullet p_{0}=\emptyset$ (there are no incoming arcs into place $\left.p_{0}\right)$. A sink place is a place $p \in P$ such that $p \bullet=\emptyset$ (there are no outgoing arcs from $p$ ). 
A Petri net structure without any specific initial marking is denoted by $P N$. A Petri net with the given initial marking is denoted by $\left(P N, M_{0}\right)$. Notice that if $W(p, q)=a$ or $W(q, p)=b$ for $a, b \in \mathbb{N}_{+}^{1}$ then this is often represented graphically by $a,(b)$ arcs from $p$ to $q$ ( $q$ to $p$ ), each with no numeric label.

Let $M_{k}\left(p_{i}\right)$ denote the marking (i.e., the number of tokens) at place $p_{i} \in P$ at time $k$, and let $M_{k}=$ $\left[M_{k}\left(p_{1}\right), \ldots, M_{k}\left(p_{m}\right)\right]^{T}$ denote the marking (state) of $P N$ at time $k$. A transition $q_{j} \in Q$ is said to be enabled at time $k$ if $M_{k}\left(p_{i}\right) \geq W\left(p_{i}, q_{j}\right)$ for all $p_{i} \in P$ such that $\left(p_{i}, q_{j}\right) \in F\left(\forall p_{i} \in \bullet q_{j}\right)$. It is assumed that at each time $k$ there exists at least one transition to fire. If a transition is enabled then it can fire. If an enabled transition $q_{j} \in Q$ fires at time $k$ then the next marking $M_{k+1}$, written as $M_{k} \stackrel{q_{j}}{\longrightarrow} M_{k+1}$, for $p_{i} \in P$ is given by

$$
M_{k+1}\left(p_{i}\right)=M_{k}\left(p_{i}\right)+W\left(q_{j}, p_{i}\right)-W\left(p_{i}, q_{j}\right) .
$$

Let $A=\left[a_{i j}\right]$ denote an $n \times m$ matrix of integers, called the incidence matrix, where $a_{i j}=a_{i j}^{+}-a_{i j}^{-}$ with $a_{i j}^{+}=W\left(q_{i}, p_{j}\right)$ and $a_{i j}^{-}=W\left(p_{j}, q_{i}\right)$. Let $u_{k} \in\{0,1\}^{n}$ denote a firing vector where if $q_{j} \in$ $Q$ is fired then its corresponding firing vector is $u_{k}=$ $[0, \ldots, 0,1,0, \ldots, 0]^{T}$ with the one in the $j$-th position in the vector and zeros everywhere else. The matrix equation (nonlinear difference equation) describing the dynamical behavior represented by a Petri net is

$$
M_{k+1}=M_{k}+A^{T} u_{k},
$$

where if at step $k, a_{i j}^{-}<M_{k}\left(p_{j}\right)$ for all $p_{j} \in P$ then $q_{i} \in Q$ is enabled, and if this $q_{i} \in Q$ fires then its corresponding firing vector $u_{k}$ is utilized in the difference equation (2) to generate the next step. Notice that if $M^{\prime}$ can be reached from some other marking $M$ and, if we fire some sequence of $d$ transitions with corresponding firing vectors $u_{0}, u_{1}, \ldots, u_{d-1}$, we obtain that

$$
M^{\prime}=M+A^{T} u, u=\sum_{k=0}^{d-1} u_{k} .
$$

Given $\sigma=q_{1}, q_{2}, \ldots, q_{n} \in Q^{*}$ (i.e., $q_{i} \in Q$ ), where $Q^{*}$ is the reflexive transitive closure of $Q$, we write $M_{0} \stackrel{\sigma}{\longrightarrow} M_{n}$ if there exist markings $M_{1}, \ldots, M_{n-1}$ such that $M_{0} \stackrel{q_{1}}{\longrightarrow} M_{1} \stackrel{q_{2}}{\longrightarrow} M_{2}, \ldots, M_{n-1} \stackrel{q_{n}}{\longrightarrow} M_{n}$. Then, we say that $M_{n}$ is reachable. The set of reachable markings of $P N$ is denoted by $R\left(P N, M_{0}\right)$, called the reachability set, and is defined by $R\left(P N, M_{0}\right)=$ $\left\{M \mid \exists \sigma \in Q^{*} M_{0} \stackrel{\sigma}{\longrightarrow} M_{k}: 0 \leq k \leq n\right\}$.

A Petri net $P N$ is $s$-bounded if $M(p) \leq s$ for every reachable marking $M$ and every place $p$ of $P N$, and bounded if it is s-bounded for some $s \geq 0$. A 1-bounded net is also called safe.

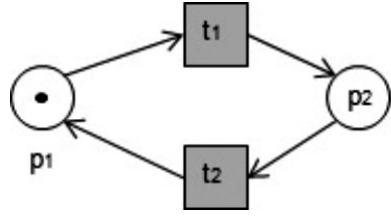

Fig. 1. Cycle.

A Petri net is strongly connected if for every two nodes $n_{1}$ and $n_{2}, n_{1}, n_{2} \in P \cup Q$, there exists a directed path leading from $n_{1}$ to $n_{2}$.

A Petri net $P N$ is a free-choice Petri net (van der Aalst, 2011) if for every two transitions $q_{i}, q_{j} \in Q, \bullet q_{i} \cap$ $\bullet q_{j} \neq \emptyset$ implies $\bullet q_{i}=\bullet q_{j}$.

Let $\left(\mathbb{N}_{+}^{n_{0}}, d\right)$ be a metric space where $d: \mathbb{N}_{+}^{n_{0}} \times$ $\mathbb{N}_{+}^{n_{0}} \rightarrow \mathbb{R}_{+}$is defined by

$$
\begin{array}{r}
d\left(M_{1}, M_{2}\right)=\sum_{i=1}^{m} \zeta_{i}\left|M_{1}\left(p_{i}\right)-M_{2}\left(p_{i}\right)\right|, \\
\zeta_{i}>0, \quad i=1, \ldots, m .
\end{array}
$$

\section{Motivation}

The main point of $P N$ is its ability to represent mark properties that involve theoretic notions of stability. In this sense, the sink (last place) of $P N$ is a place whose marking is bounded and it does not change. Therefore, two main concepts must be considered carefully within the notion of stability: cycle and block.

The $P N$ shown in Fig. 1 represents a cycle. It has the property of stability, because given the incidence matrix

$$
A=\left[\begin{array}{cc}
-1 & 1 \\
1 & -1
\end{array}\right]
$$

and picking the positive vector $\Phi=\left[\begin{array}{ll}2 & 2\end{array}\right]>0$ since $A$ is already the transpose, we obtain that $A \Phi^{T}=\left[\begin{array}{ll}0 & 0\end{array}\right] \leq 0$ (concluding stability). But the $P N$ has no final place.

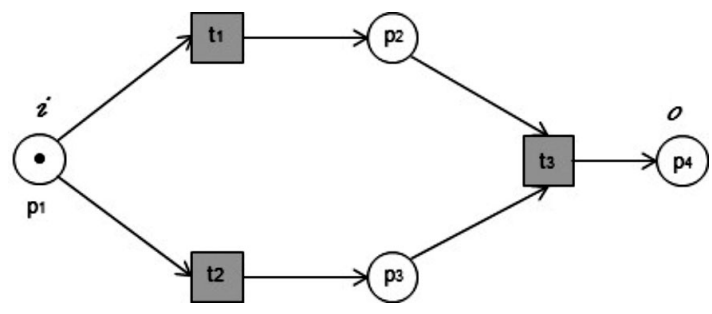

Fig. 2. Block.

The $P N$ represented in Fig. 2 represents a block. It has the property of stability, because the incidence matrix

$$
A=\left[\begin{array}{cccc}
-1 & 1 & 0 & 0 \\
-1 & 0 & 1 & 0 \\
0 & -1 & -1 & 1
\end{array}\right]
$$


and picking the positive vector such that $\Phi=$ $\left[\begin{array}{llll}2 & 1 & 1 & 1\end{array}\right]>0$ since $A$ is already the transpose, we obtain that $A \Phi^{T}=\left[\begin{array}{lll}-1 & -1 & -1\end{array}\right] \leq 0$ (concluding stability). But, the sink of the $P N$ never can be reached.

Loosely speaking, a workflow net is a Petri net with two distinguished input and output places without input and output transitions, respectively, and such that the addition of a reset transition leading back from the output to the input place makes the net strongly connected. Formally, we have the following.

Definition 1. A Petri net $P N=(P, Q, F, W, M)$ is a workflow net if

- there exist places $i, o \in P$ such that $\bullet i=\emptyset=o \bullet$, $M(p)=1$ for $p=i$ and $M(p)=0$ otherwise,

- every node is in a path from $i$ to $o$, i.e., for any $x \in$ $P \cup Q:(i, x) \in F^{*}$ and $(x, o) \in F^{*}$, where $F^{*}$ is the reflexive-transitive closure of relation $F$.

Then, the resulting Petri net is strongly connected. A workflow net $P N$ is sound if it is live and bounded (cf. van der Aalst, 1998; 2011).

Definition 2. Let $P N$ be a workflow net. $P N$ is sound if the following three requirements are satisfied:

1. For every state $M$ reachable from state $M_{i}$, there exists a firing sequence leading from state $M$ to state $M_{o}$ :

$$
\text { for all } M:\left(M_{i} \stackrel{\sigma}{\rightarrow} M\right) \Rightarrow\left(M \stackrel{\sigma}{\rightarrow} M_{o}\right) \text {. }
$$

2. State $M_{o}$ is the only state reachable from state $M_{i}$ with at least one token in place $M_{O}$ :

$$
\text { for all } M:\left(M_{i} \stackrel{\sigma}{\rightarrow} M \wedge M \geq 0\right) \Rightarrow\left(M=M_{o}\right) \text {. }
$$

3. There are no dead transitions in $P N$ :

for all $q \in Q$, there exist $M, M \prime$ :

$$
\left(M_{i} \stackrel{\sigma}{\rightarrow} M \stackrel{q}{\rightarrow} M \prime\right) .
$$

The first requirement states that, starting from the initial state $M_{i}$, it is always possible to reach the state with one token in place $o$. The second requirement states that, the moment a token is put in place $o$, all the other places should be empty. The third requirement has been added to avoid activities and conditions which do not contribute to the processing of cases. Although it is the looked-for soundness of workflow nets, many of the real models with conditional behavior will not satisfy the third requirement: "no dead transitions" in $P N$. The problem is usually produced by the operations needed to be modeled and not necessarily by the structure of the net. In this sense, a workflow satisfies the soundness property if, given its corresponding Petri net (finite and non-blocking), which is tracked forward, provided one starts with a single token in the source and regardless of how the computation proceeds at start, it is always possible to reach a state with the token in the sink place.

Definition 3. Let $P N$ be a workflow net. $P N$ is weak sound if the following two requirements are satisfied:

1. For every state $M$ reachable from state $M_{i}$, there exists a firing sequence leading from state $M$ to state $M_{o}$ :

$$
\text { for all } M:\left(M_{i} \stackrel{\sigma}{\rightarrow} M\right) \Rightarrow\left(M \stackrel{\sigma}{\rightarrow} M_{o}\right) \text {. }
$$

2. State $M(o)$ is the only state reachable from state $M_{i}$ with at least one token in place $M_{o}$ :

$$
\text { for all } M:\left(M_{i} \stackrel{\sigma}{\rightarrow} M \wedge M \geq 0\right) \Rightarrow\left(M=M_{o}\right) \text {. }
$$

Soundness requires that a workflow net be always able to terminate in the sink of $P N$. Therefore, if we want to use stability as a theoretic notion for finding the soundness of a workflow net, it will be required to impose two conditions over the corresponding $P N$ : finite (no cycles) and non-blocking.

\section{Workflow soundness property}

Let us consider systems of first ordinary difference equations given by

$$
\begin{aligned}
x(n+1) & =\psi[n, x(n)], \\
x\left(n_{0}\right) & =x_{0} .
\end{aligned}
$$

for $n \in \mathbb{N}_{+}^{n_{0}}$, where $x(n) \in \mathbb{R}^{d}$ and $\psi: \mathbb{N}_{+}^{n_{0}} \times \mathbb{R}^{d} \rightarrow \mathbb{R}^{d}$ is continuous in $x(n)$.

Definition 4. The $n$-vector valued function $\phi\left(n, n_{0}, x_{0}\right)$ is a solution of (5) if $\phi\left(n_{0}, n_{0}, x_{0}\right)=x_{0}$ and $\phi(n+$ $\left.1, n_{0}, x_{0}\right)=\psi\left(n, \phi\left(n, n_{0}, x_{0}\right)\right)$ for all $n \in \mathbb{N}_{+}^{n_{0}}$.

Definition 5. The system (5) is said to be practically stable (Lakshmikantham and Martynyuk, 1990; Lakshmikantham et al., 1991) if, given $(\lambda, \Psi)$ with $0<$ $\lambda<\Psi$, we have that

$$
\begin{aligned}
\left|x_{0}\right|<\lambda \Rightarrow\left|x\left(n, n_{0}, x_{0}\right)\right| & <\Psi, \\
& \forall n \in \mathbb{N}_{+}^{n_{0}}, \quad n_{0} \geq 0 .
\end{aligned}
$$

Definition 6. (Lakshmikantham and Martynyuk, 1990; Lakshmikantham et al., 1991) The system (5) is said to be uniformly practically stable if it is practically stable for every $n_{0} \geq 0$.

Definition 7. A continuous function $\alpha:[0, \infty) \rightarrow[0, \infty)$ is said to belong to the class $\mathcal{K}$ if it is strictly increasing and $\alpha(0)=0$. 
Let us consider (Lakshmikantham et al., 1991) the vector function $v(n, x(n)), v: \mathbb{N}_{+}^{n_{0}} \times \mathbb{R}^{d} \rightarrow \mathbb{R}_{+}^{p}$, and let us define the variation of $v$ relative to $(5)$ by

$$
\Delta v=v(n+1, x(n+1))-v(n, x(n)) .
$$

Then we have the following results due to Lakshmikantham and Martynyuk (1990), Lakshmikantham et al. (1991) as well as Passino et al. (1995).

Theorem 1. Let $v: \mathbb{N}_{+}^{n_{0}} \times \mathbb{R}^{n} \rightarrow \mathbb{R}_{+}$be a continuous function in $x$, such that for $\beta, \alpha \in \mathcal{K}$ we have $\beta(|x|) \leq v(n, x(n)) \leq \alpha(|x|)$ and $\Delta v(n, x(n)) \leq$ $w(n, v(n, x(n)))$ holds for $n \in \mathbb{N}_{+}^{n_{0}}, x(n) \in \mathbb{R}^{n}$, where $w: \mathbb{N}_{+}^{n_{0}} \times \mathbb{R}_{+} \rightarrow \mathbb{R}$ is a continuous function in the second argument. Let us suppose that $\gamma(n, u) \equiv u+w(n, u)$ is non-decreasing in $u, 0<\lambda<\Psi$ are given and finally that $\alpha(\lambda)<\beta(\Psi)$ is satisfied. Then the stability properties of

$$
u(n+1)=\gamma(n, u(n)), u\left(n_{0}\right)=u_{0} \geq 0
$$

imply the corresponding stability properties of the system (5).

We will extend Theorem 1 to the case of several Lyapunov functions. Let us consider a vector Lyapunov function $v(n, x(n)), v: \mathbb{N}_{+}^{n_{0}} \times \mathbb{R}^{d} \rightarrow \mathbb{R}_{+}^{p}$, and let us define the variation of $v$ relative to (5). Then, we have the following theorem (Lakshmikantham et al., 1991).

Theorem 2. Let $v: \mathbb{N}_{+}^{n_{0}} \times \mathbb{R}^{d} \rightarrow \mathbb{R}_{+}^{p}$ be a continuous function in $x$, and define the function $v_{0}(n, x(n))=$ $\sum_{i=1}^{p} v_{i}(n, x(n))$ such that it satisfies the estimates

$$
\beta(|x|) \leq v_{0}(n, x(n)) \leq \alpha(|x|)
$$

for $\alpha, \beta \in \mathcal{K}$ and

$$
\Delta v(n, x(n)) \leq w(n, v(n, x(n)))
$$

for $n \in \mathbb{N}_{+}^{n_{0}}, x(n) \in \mathbb{R}^{d}$, where $w: \mathbb{N}_{+}^{n_{0}} \times \mathbb{R}_{+}^{p} \rightarrow \mathbb{R}^{p}$ is a continuous function in the second argument. Assume that $\gamma(n, u) \doteq q u+w(n, u)$ is non decreasing in $u, 0<$ $\lambda<\Psi$ are given and $\alpha(\lambda)<\beta(\Psi)$ is satisfied. Then the practical stability properties of

$$
u(n+1)=\gamma(n, u(n)), \quad u\left(n_{0}\right)=u_{0} \geq 0
$$

imply the corresponding practical stability properties of the system (5).

Then, we have the following result (Lakshmikantham et al., 1991).

Corollary 1. From Theorem 2 we have the following:

1. If $w(n, e) \equiv 0$, we obtain uniform practical stability of (5), which implies structural stability (Lakshmikantham et al., 1991).
2. If $w(n, e)=-c(e)$ for $c \in \mathcal{K}$, then we obtain uniform practical asymptotic stability of (5), (cf. Lakshmikantham et al., 1991).

For Petri nets we have the following results of stability (Passino et al., 1995).

Proposition 1. Let $P N$ be a Petri net. Therefore, $P N$ is uniform practical stable if there exists a $\Phi$ strictly positive $m$ vector such that

$$
\Delta v=u^{T} A \Phi \leq 0 .
$$

Moreover, $P N$ is uniform practical asymptotic stability if the following equation holds:

$$
\Delta v=u^{T} A \Phi \leq-c(e), \quad c \in \mathcal{K} .
$$

Proof. Let us choose as our candidate Lyapunov function $v(M)=M^{T} \Phi$ with $\Phi$ being an $m$ vector to be chosen. It is simple to verify that $v$ satisfies all the conditions of Theorem 2. Therefore, the uniform practical asymptotic stability is obtained if there exists a strictly positive vector $\Phi$ such that Eqn. (12) holds.

Proposition 2. Let $P N$ be a Petri net. Therefore, $P N$ is uniformly practically stable if there exists a strictly positive $m$ vector $\Phi$ such that

$$
\Delta v=u^{T} A \Phi \leq 0 \Leftrightarrow A \Phi \leq 0 .
$$

Proof.

(Necessity) Since $u^{T} A \Phi \leq 0$ holds, for every $u$ we have that $A \Phi \leq 0$.

(Sufficiency) This results from the fact that $u$ is positive.

Remark 1. The 'if an only if' relationship of (14) results from the fact that $u$ is positive.

We have the following theorem that characterizes the soundness property.

Theorem 3. Let $P N$ be a finite and non-blocking workflow net. Then, $P N$ satisfies the soundness property iff there exists a strictly positive $m$ vector $\Phi$ such that $\Delta v=u^{T} A \Phi \leq 0$

Proof.

(Necessity) It follows directly from Proposition 1 and Proposition 2,

(Sufficiency) Let us suppose by contradiction that $u^{T} A \Phi>0$ with $\Phi$ fixed. From $M^{\prime}=M+u^{T} A$ we have that $M^{\prime} \Phi=M \Phi+u^{T} A \Phi>M \Phi$. Then it is possible to construct an increasing sequence $M \Phi<$ $M^{\prime} \Phi<\ldots<M^{n} \Phi<\ldots$ which grows up without any bound. Therefore, the $P N$ is not uniformly practically stable. 


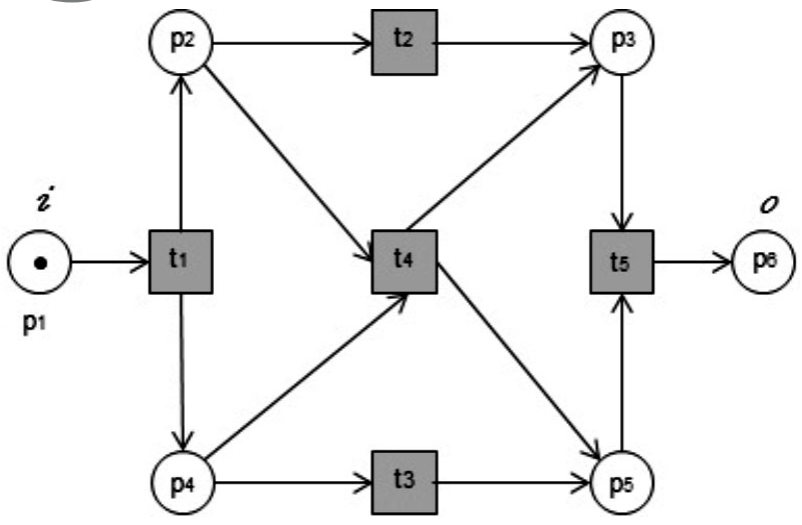

Fig. 3. Workflow net that is sound.

Remark 2. The finite and non-blocking conditions over the workflow net cannot be relaxed (see Section 3) and reinforce the definition of the workflow (Definition 2):

1. If the workflow is into a cycle, it will satisfy the theoretic notion of stability, but it will never reach the sink place of the net. If we required termination without this assumption, all nets allowing loops in their execution sequences would be called unsound, which is clearly not desirable.

2. If we suppose that the workflow net blocks at some place $p$, it will also satisfy the theoretic notion of stability, but it will never reach the sink place of the net.

\section{Application examples}

The aim of this section is to present application examples represented by a workflow concluding soundness.

Example 1. In the Petri net shown in Fig. 3 only one place is initially marked. Here $t_{1}$ is enabled and the firing of $t_{1}$ will result in the state that marks places $p_{2}$ and $p_{4}$. In this state, $t_{2}, t_{3}$ and $t_{4}$ are enabled. If $t_{2}$ fires, $t_{4}$ becomes disabled, but $t_{3}$ remains enabled. Similarly, if $t_{3}$ fires, $t_{4}$ becomes disabled, but $t_{2}$ remains enabled, etc. The incidence matrix of the workflow net shown in Fig. 3 is given by

$$
A=\left[\begin{array}{cccccc}
-1 & 1 & 0 & 1 & 0 & 0 \\
0 & -1 & 1 & 0 & 0 & 0 \\
0 & 0 & 0 & -1 & 1 & 0 \\
0 & -1 & 1 & -1 & 1 & 0 \\
0 & 0 & -1 & 0 & -1 & 1
\end{array}\right]
$$

and picking the positive vector

$$
\Phi=\left[\begin{array}{llllll}
4 & 2 & 1 & 1 & 1 & 1
\end{array}\right]>0
$$

since $A$ is already the transpose, we obtain that

$$
A \Phi^{T}=\left[\begin{array}{lllll}
-1 & -1 & 0 & -1 & -1
\end{array}\right] \leq 0,
$$

concluding soundness (stability).

If we remove transition $t_{4}$, the resulting net is a free-choice Petri net. These types of Petri nets are interesting from the viewpoint of analysis (van der Aalst, 2011): (i) liveness and boundedness can be decided in polynomial time for free-choice nets (this is not the case for non-free-choice Petri nets) and (ii) they always satisfy the soundness properties.

Example 2. Let us consider an insurance broker agency. As a broker, the agency sells policies for different companies. The main products are life and automobile policies. For selling and advertising, the insurance company obtains detailed information from potential customers, as well as private and governmental agencies. This information is distributed between the company's agents who contact potential clients via phone and try to set up a conference call. However, they also have their own sources of information. At the interview, the agent examines the client's current insurance coverage and tries to find an opportunity for a policy that will best fit the customer's needs.

Before obtaining an insurance policy, the new client must undergo an identity investigation. In the case of a life insurance, the client has, in addition, to approve a physical examination test in an accredited hospital. If the investigation is positive, both the parts sign a policy and keep a copy of the contract. If, during the investigation, irregularities are found, the agent is informed and meets the client in order to find new options.

The insurance policy is in effect when the client makes the first insurance premium payment. Every policy carries with a schedule of premiums, which varies with the type and coverage. Each policy provides a commission for the agency. The commission varies with the insurance company, policy type and coverage. The insurance company management defines the commission policy, which varies from agency to agency. The agency splits the commission received for each policy with the agent who sold it. The rate depends on the seniority of the agent. Once a policy has been sold, the agency submits premium bills to the client, collects payment and sends the payment, minus the commission, to the insurance company.

If a client fails to pay premiums, the agent who sold the policy is informed, so that they can contact the client. Claims can be made on insurance policies as specified in the policy itself. Clients or beneficiaries contact the agent to file such claims. Life insurance claims may be made by the beneficiaries on the death of the insured. In both the cases, the insurance company sends an adjuster to legitimate the claim and arrange the final insurance details. For an automobile insurance policy, claims are made when the car is involved in an accident, damaged or stolen. For simplification, we will consider just the 


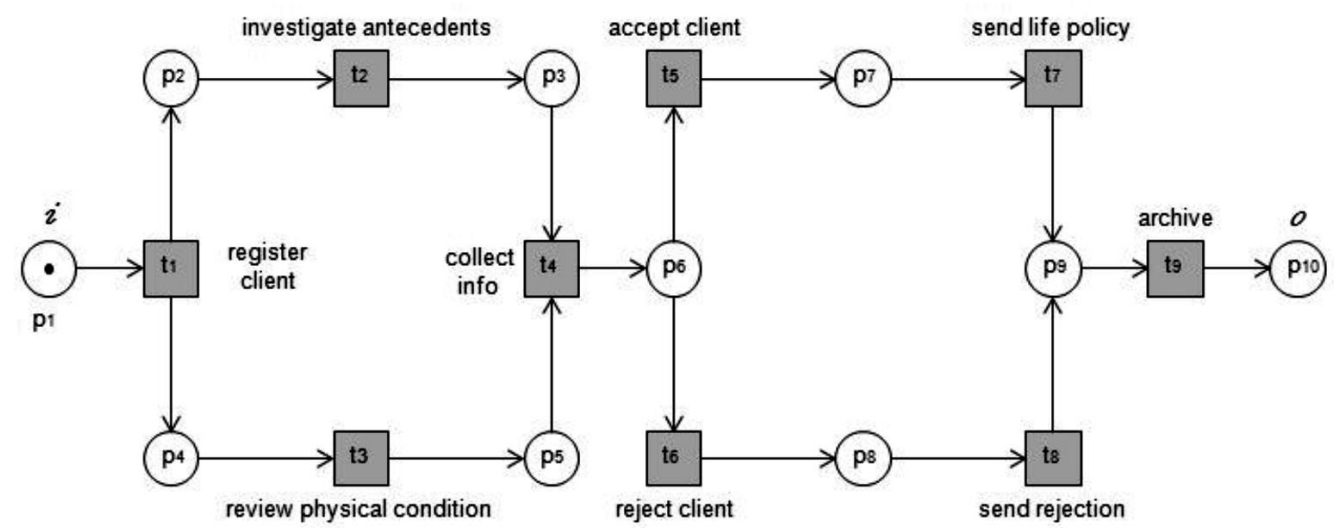

Fig. 4. Insurance broker agency workflow net.

organizational strategy of the insurance company.

The insurance broker agency business process is represented in Fig. 4 by a free-choice $P N$. (It is important to note that the $P N$ represented in Fig. 4 is a simplification of the workflow explained in the text description of the broker agency routines.) Now, the incidence matrix $A$ of the workflow net shown in Fig. 4 is given by

$$
\left[\begin{array}{cccccc}
-1 & 1 & 0 & 1 & 0 & \\
0 & -1 & 1 & 0 & 0 & \\
0 & 0 & 0 & -1 & 1 & \\
0 & 0 & -1 & 0 & -1 & \\
0 & 0 & 0 & 0 & 0 & \\
0 & 0 & 0 & 0 & 0 & \\
0 & 0 & 0 & 0 & 0 & \\
0 & 0 & 0 & 0 & 0 & \\
0 & 0 & 0 & 0 & 0 & \\
& 0 & 0 & 0 & 0 & 0 \\
& 0 & 0 & 0 & 0 & 0 \\
& 0 & 0 & 0 & 0 & 0 \\
& 1 & 0 & 0 & 0 & 0 \\
& -1 & 1 & 0 & 0 & 0 \\
& -1 & 0 & 1 & 0 & 0 \\
& 0 & -1 & 0 & 1 & 0 \\
& 0 & 0 & -1 & 1 & 0 \\
& 0 & 0 & 0 & -1 & 1
\end{array}\right]
$$

and picking the positive vector

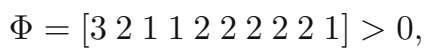

since $A$ is already the transpose, we obtain that

$$
A \Phi^{T}=[0-1-1-100000-1] \leq 0,
$$

concluding soundness (stability).

\section{Conclusion and future work}

Reasoning about the correctness of a workflow model without any domain knowledge corresponds to the soundness (soundness) property. A workflow net satisfies the soundness property if its Petri net representation is tracked forward from its source place and a natural form of termination is ensured by a sink. This paper provided an analytical method for solving the soundness property verification problem. The method is useful for practical applications and guarantees that anomalies can be detected without domain knowledge. To present our statement, we used Lyapunov stability theory, concluding that if a workflow net is stable then it satisfies the soundness property. This method can be easily implemented into existing commercial systems that do not support the verification of workflows.

It is important to note that the key contribution of the paper is the analytical method itself. The definition of soundness is introduced because the proposed method only satisfies part of the soundness property (van der Aalst, 2011). In this sense, the proposed analytical method is a step forward in checking the soundness of workflow nets.

Without doubt there are more than a few theoretical challenges that need to be considered in future research in Lyapunov-based theory for solving the soundness verification problem. This paper has interesting implications for using more sophisticated definitions of Petri nets, because the Lyapunov method introduces new concepts in the Petri nets area. In this work, we consider dynamical systems governed by ordinary difference equations described by Petri nets. Then, an important emerging open research challenge is the use of Lyapunov theory to produce a trajectory tracking function (Lyapunov-like function) as a solution to the difference equation (constructed with respect the constraints imposed by the system). Then the Lyapunov-like function will compute the trajectory of the token over the Petri net, converging naturally into the sink place (Clempner, 2005). 


\section{References}

Barkaoui, K. and Ben Ayed, R. (2011). Uniform verification of workflow soundness, Transactions of the Institute of Measurement and Control 33(1): 133-148.

Barkaoui, K. and Petrucci, L. (1998). Structural analysis of workflow nets with shared resources, Proceedings of Workflow Management: Net-based Concepts, Models, Techniques and Tools (WFM'98), Lisbon, Portugal, pp. 82-95.

Basu, A. and Blanning, R.W. (2000). A formal approach to workflow analysis, Information Systems Research 11(1): $17-36$

Basu, A. and Blanning, R.W. (2002). Research commentary: Workflow management issues in e-business, Information Systems Research 13(1): 17-36.

Bi, H.H. and Zhao, J.L. (2004). Applying propositional logic to workflow verification, Information Systems Research 5(3-4): 293-318.

Brams, G.W. (1983). Réseaux de Petri: Théorie et Pratique, Théorie et Analyse (Petri Nets: Theory and Practice), Vol. 1, Masson, Paris.

Clempner, J. (2005). Colored decision process Petri nets: Modeling, analysis and stability, International Journal of Applied Mathematics and Computer Science 15(3): 405-420.

Clempner, J. (2014). Verifying soundness of business processes: A decision process Petri nets approach, Expert Systems with Applications 41(11): 5030-5040.

Clempner, J. and Retchkiman, Z. (2005). Business process modeling using decision process Petri nets, WSEAS Transactions on Business and Economics 2(2): 36-44.

Dehnert, J. and Rittgen, P. (2001). Relaxed soundness of business processes, in K.R. Dittrich, A. Geppert and M.C. Norrie (Eds.), Proceedings of the 13th International Conference on Advanced Information Systems Engineering (CAiSE'01), Lecture Notes in Computer Science, Vol. 2068, Springer-Verlag, Berlin, pp. 157-170.

Desel, J. and Erwin, T. (2000). Modeling, simulation and analysis of business processes, in W.M.P. van der Aalst, J. Desel and A. Oberweis (Eds.), Business Process Management: Models, Techniques and Empirical Studies, Lecture Notes in Computer Science, Vol. 1806, Springer-Verlag, London, pp. 129-141.

Ellis, C.A. and Nutt, G.I. (1993). Modelling and enactment of workflow systems, in M. Ajmone Marsan (Ed.), 14th International Conference on Application and Theory of Petri Nets (ICATPN 1993), Lecture Notes in Computer Science, Vol. 691, Springer-Verlag, Berlin, pp. 1-16.

Fu, X., Bultan, T. and Su, J. (2002). Formal verification of e-services and workflows, in C. Bussler, R. Hull, S. McIlraith, M. Orlowska, B. Pernici, and J. Yang (Eds.), Web Services, E-Business, and the Semantic Web, CAiSE 2002 International Workshop (WES 2002), Lecture Notes in Computer Science, Vol. 2512, Springer-Verlag, Berlin, pp. 188-202.
Fu, X., Bultan, T. and Su, J. (2004). Analysis of interacting BPEL web services, 13th International World Wide Web Conference, New York, NY, USA, pp. 621-630.

Karamanolis, C., Giannakopoulou, D., Magee, J. and Wheater, S.M. (2000). Model checking of workflow schemas, Proceedings of the 4th International Enterprise Distributed Object Computing Conference (EDOC'O0), Los Alamitos, CA, USA, pp. 170-181.

Kindler, E., Martens, A. and Reisig, W. (2000). Inter-operability of workflow applications: Local criteria for global soundness, in W.M.P. van der Aalst, J. Desel and A. Oberweis (Eds.), Business Process Management: Models, Techniques, and Empirical Studies, Lecture Notes in Computer Science, Vol. 1806, Springer-Verlag, Berlin, pp. 235-253.

Lakshmikantham, V., Leela, S. and Martynyuk, A.A. (1990) Practical Stability of Nonlinear Systems, World Scientific, Singapore.

Lakshmikantham, V., Matrosov, V.M. and Sivasundaram, S. (1991). Vector Lyapunov Functions and Stability Analysis of Nonlinear Systems, Kluwer Academic Publishers, Dordrecht.

Lin, H., Zhao, Z., Li, H. and Chen, Z. (2002). A novel graph reduction algorithm to identify structural conflicts, Proceedings of the 34th Annual Hawaii International Conference on System Science (HICSS-35), Maui, HI, USA, pp. 1-10.

Lohmann, N., Massuthe, P., Stahl, C. and Weinberg, D. (2006). Analyzing interacting BPEL processes, in S. Dustdar, J.L. Fiadeiro and A. Sheth (Eds.), International Conference on Business Process Management (BPM 2006), Lecture Notes in Computer Science, Vol. 4102, Springer-Verlag, Berlin, pp. 17-32.

Mann, Z.A. (2010). Numbering action vertices in workflow graphs, International Journal of Applied Mathematics and Computer Science 20(3): 591-600, DOI: 10.2478/v10006-010-0044-0.

Martens, A. (2005a). Analyzing web service based business processes, in M. Cerioli (Ed.), Proceedings of the 8th International Conference on Fundamental Approaches to Software Engineering (FASE 2005), Lecture Notes in Computer Science, Vol. 3442, Springer-Verlag, Berlin, pp. 19-33.

Martens, A. (2005b). Consistency between executable and abstract processes, Proceedings of the International IEEE Conference on e-Technology, e-Commerce, and e-Services (EEE'05), Kitakyushu, Japan, pp. 60-67.

Mendling, J., Neumann, G. and van der Aalst, W.M.P. (2007). Understanding the occurrence of errors in process models based on metrics, in M. Cerioli (Ed.), Proceedings of the OTM Conference on Cooperative information Systems (CoopIS 2007), Lecture Notes in Computer Science, Vol. 4803, Springer-Verlag, Berlin, pp. 113-130.

Murata, T. (1989). Petri nets: Properties, analysis and applications, Automatica 77(4): 541-580. 
Passino, K.M., Burguess, K.L. and Michel, A.N. (1995). Lagrange stability and boundedness of discrete event systems, Theory and Applications 5: 383-403.

Sadiq, W. and Orlowska, M.E. (1997). On correctness issues in conceptual modeling of workflows, Proceedings of the 5th European Conference on Information Systems (ECIS '97), Cork, Ireland, pp. 19-21.

Sadiq, W. and Orlowska, M.E. (2000). Analyzing process models using graph reduction techniques, Information Systems 25(2): 117-134

Salimifard, K. and Wright, M. (2001). Petri net-based modelling of workflow systems: An overview, European Journal of Operational Research 134(3): 664-676.

van der Aalst, W.M.P. (1997). Verification of workflow nets, in P. Azéma and G. Balbo (Eds.), Application and Theory of Petri Nets 1997, Springer-Verlag, Berlin, pp. 407-426.

van der Aalst, W.M.P. (1998). The application of Petri nets to workflow management, The Journal of Circuits, Systems and Computers 8(1): 21-66.

van der Aalst, W.M.P. (2007). Workflow verification: Finding control-flow errors using Petri-net-based techniques, in W.M.P. van der Aalst, J. Desel and A. Oberweis (Eds.), Business Process Management: Models, Techniques, and Empirical Studies, Springer-Verlag, Berlin, pp. 161-183.

van der Aalst, W.M.P. (2011). Soundness of workflow nets: Classification, decidability, and analysis, Formal Aspects of Computing 23(3): 333-363.

van Dongen, B.F., van der Aalst, W.M.P. and Verbeek, H.M.W. (2005). Verification of EPCs: Using reduction rules and Petri nets, in O. Pastor and J. Falco e Cunha (Eds.), Proceedings of the 17th Conference on Advanced Information Systems Engineering (CAiSE'05), Lecture Notes in Computer Science, Vol. 3520, Springer-Verlag, Berlin, pp. 372-386.

van Hee, K.M., Serebrenik, A., Sidorova N. and Voorhoeve, M. (2005). Soundness of resource-constrained workflow nets, in G. Ciardo and P. Darondeau (Eds.), Applications and Theory of Petri Nets 2005, Lecture Notes in Computer Science, Vol. 3536, Springer-Verlag, Berlin, pp. 250-267.

van Hee, K.M., Sidorova, N. and Voorhoeve, M. (2004). Generalised soundness of workflow nets is decidable, in J. Cortadella and W. Reisig (Eds.), Application and Theory of Petri Nets 2004, Lecture Notes in Computer Science, Vol. 3099, Springer-Verlag, Berlin, pp. 197-215.

Vanhatalo, J., Volzer, H. and Leymann, F. (2007). Faster and more focused control-flow analysis for business process models through SESE decomposition, in B. Kramer, K. Lin and P. Narasimhan (Eds.), Proceedings of Service-Oriented Computing (ICSOC 2007), Lecture Notes in Computer Science, Vol. 4749, Springer-Verlag, Berlin, pp. 43-55.

Verbeek, H.M.W., Basten, T. and van der Aalst, W.M.P. (2001). Diagnosing workflow processes using Woflan, The Computer Journal 44(4): 246-279.

Verbeek, H.M.W., van der Aalst W.M.P. and ter Hofstede, A.H.M. (2001). Verifying workflows with cancellation regions and or-joins: An approach based on relaxed soundness and invariants, The Computer Journal 50(3): 294-314.

Weske, M. (2007). Business Process Management: Concepts, Languages, Architectures, Springer-Verlag, Berlin.

Wombacher, A. (2006). Decentralized consistency checking in cross-organizational workflows, Proceedings of the International Conference on e-Technology, e-Commerce and e-Service (CEC/EEE 2006), Washington, DC, USA, pp. 39-46.

Wynn, M.T., Edmond, D., van der Aalst W.M.P. and ter Hofstede, A.H.M. (2005). Achieving a general, formal and decidable approach to the or-join in workflow using reset nets, in G. Ciardo and P. Darondeau (Eds.), Applications and Theory of Petri Nets 2005, Lecture Notes in Computer Science, Vol. 3536, Springer-Verlag, Berlin, pp. 423-443.

Wynn, M.T., van der Aalst, W.M.P., ter Hofstede, A.H.M. and Edmond, D. (2006). Verifying workflows with cancellation regions and or-joins: An approach based on reset nets and reachability analysis, in S. Dustdar, J.L. Fiadeiro and A. Sheth (Eds.), International Conference on Business Process Management (BPM 2006), Lecture Notes in Computer Science, Vol. 4102, Springer-Verlag, Berlin, pp. 389-394.

zur Muehlen, M. (2004). Workflow-based Process Controlling: Foundation, Design and Application of Workflow-driven Process Information Systems, Logos, Berlin.

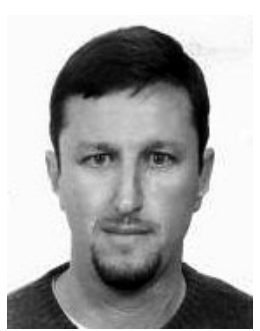

Julio Clempner has more than ten years of experience in the field of management consulting. Currently, he specializes in the application of high technology. He has also conducted projects related to IT government, project management, analysis and design of software (RUP, UML), development of software (add-hoc and products), information technology strategic planning, balanced scorecard, evaluation of software and business process reengineering (SIX-SIGMA) for infusing advanced computing technologies into diverse lines of businesses. Dr Julio Clempner is now involved in providing e-business solutions, including e-business strategy, management of technology, operations strategy, project management, continuous quality improvement, and managing total quality transformation. Dr. Clempner's research interests are focused on justifying and introducing the Lyapunov equilibrium point in shortest-path decision processes and shortest-path game theory, which has lead to several streams of research. Dr. Julio Clempner is a member of the Mexican National System of Researchers (SNI).

Received: 4 July 2013

Revised: 30 November 2013

Re-revised: 24 February 2014 